Pag-usbong ng balbal na pananalita bilang modernong wika ng kabataan: Isang pagsusuri

Noval, Arnel T.

Cebu Techological University, Cebu, Philippines (arnelnoval663@gmail.com)

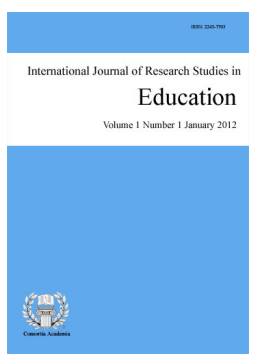

ISSN: 2243-7703 Online ISSN: 2243-7711

OPEN ACCESS

\title{
Abstract
}

This study analyzed the structure and the reasons as to how slang language emerged at present time. This also sought to find out the influences which have big impact to the emergence of slang as modern language in this generation. The data were gathered through interviewing thirteen (13) participants and interpreted through descriptive phenomenological method of research. The study revealed that the most common structure in the formation of slang words is through combination. With this, slang words were used for entertainment, self-expression and personal identity, to be in trend and for socialization. It can also be seen from the study that technology, gadget, social media, peers and friends and the artists being idolized have a big impact in the emergence of slang words. As a conclusion, the emergence of slang words in the modern generation is related to the language situation existing in a society and as to how its speakers embrace and accept the language.

Keywords: pag-usbong; balbal na pananalita; wika; kabataan; pagsusuri 


\section{Pag-usbong ng balbal na pananalita bilang modernong wika ng kabataan: Isang pagsusuri}

\section{Introduksiyon}

Sinasabi ng maraming mananaliksik at dalubwika na ang wika ay dinamiko. Umaalinsabay ito sa mabilis na pag-inog ng mundo sang-ayon sa mga pangangailangan ng lipunan at ng sitwasyong pangwika. Sa makabagong henerasyon kung saan bahagi na ng sistema ang teknolohiyang dihital, hindi maikakaila ang katotohanang napakarami na ng mga salitang nagsusulputan at nagiging uso. Ang nakatutuwa pa rito, ginagamit ito ng nakararami at halos bukambibig sa pang-araw-araw na pakikisalamuha. Ganito kalaki ang naging impluwensiya ng teknolohiya gaya ng radio, telebisyon, social media at iba pa sa buhay ng tao sa paggamit ng makabagong salitang balbal na umuusbong sa kasalukuyang panahon.

Ang salitang balbal ay tinatawag ding slang sa Ingles. Nanggagaling ang mga salitang ito sa mga grupo ng mga tao na ginagamit ang mga salita upang magkaroon ng sariling codes. Dagdag pa nila, ang isang wika ay maaaring nadaragdagan ng mga bagong bokabularyo bunga ng pagiging malikhain ng mga tao, maaaring sila ay nakalilikha ng mga bagong salita. Ang pinakamahusay na halimbawa nito ay ang mga salitang balbal at pangkabataang pananalita (Aldaca at Villarin, 2012).

Ayon naman kay Ocampo (2013), ang salitang balbal ay naging bahagi na ng bokabularyong Filipino na tumutugon sa kinakailangan ng lipunan. Masasabing ang wikang ito ay pampersonal na pakikipagkomunikasyon at tinatawag ding "salitang kalye" (halaw sa Estanislao et al., 2018). Binanggit naman nina Kazuhiro et al. (2009) halaw sa Quia et al., (2018), ang balbal na salita ay ang di pamantayang paggamit ng mga salita sa isang wika ng isang partikular na grupo ng lipunan. Isang lipunan na lalong nanghimok sa kabataan na makasanayang bigkasing madalas ang mga salitang ito gaya ng lipunang mabubuo sa social media, isang lipunang dihital na nabibigyan ang sinoman ng pagkakataong makilahok o di kaya ay makibahagi sa anomang paksa o usaping pinag-uusapan.

Ang paglaganap ng mga salitang balbal sa modernong panahon ay dulot na rin ng mga sitwasyong pangwikang dinadanas ng tagapagsalita kasangkot ang malikhaing pagpapahayag ng mga kaisipan at mga ideya patungkol sa isang tiyak na paksa. Ang malikhaing pagpapahayag na ito ay sinangkapan ng malikhaing paghahabi ng mga salita sa makabuluhang pamamaraan sa pagbuo ng mga salitang balbal sa iba't ibang henerasyon.

Sa kasalukuyang panahon, maraming wika ang nagsusulputan sang-ayon sa kung paano ito ginagamit ng lipunan. Maraming uri ng wika ang nabubuo gaya ng bekimon, conyo, gay lingo at iba pa. Ang mga wikang ito ay ang mga wikang gamit ng mga mag-aaral na kung hindi masasabayan ay magiging malaking hadlang sa larangan ng komunikasyon hindi lamang sa tahanan pati na rin sa paaralan. Kaya, nararapat lamang na maiangkop at magkaroon ng sapat na kaalaman sa mga wikang ito nang sa gayon maiiwasan ang kung anomang hindi pagkakaunawaan. Ang pag-unawa at pagkakaroon ng sapat na kaalaman sa mga balbal na pananalita ay magtutulay sa agwat ng wikang gamit ng noon at ng wikang gamit ng mga kabataan na itinatampok ng kanilang henerasyon.

Ang mananaliksik, isang guro na nagtuturo ng sabjek na Filipino ay nagnanais na matumbok ang mga aspekto ng pagsibol ng makabagong salitang balbal na karaniwang ginagamit ng kanyang mga estudyante. Sa ganitong paraan, lubusang mauunawaan ng pangkalahatan ang kanilang mga lunggati, lungkot, kabiguan at iba pang damdamin o saloobing dinaranas. Dagdag pa nito, mapalalawak ang kaalaman at pang-unawa sa paraan ng pagpapahayag ng kabataan sa kasalukyan, ang kanilang pakikisalamuha o pakikisama maging ang kanilang pag-uugali. Ito ang nagtulak sa mananaliksik upang isagawa ang pag-aaral. Sa ganitong paraan, maisusulong ang pagkakaunawaan at makadaragdag sa kaalaman sa wikang gamit ng kasalukuyan tungo sa pagpapayaman ng 
diskursong Pilipino.

Nilayon din ng pag-aaral na ito na masuri ang kadahilanan ng pagsibol ng mga makabagong salitang balbal, damdaming nararamdaman at naghimok o nagganyak sa mga kabataan bilang tagapagsalita nito na gamitin ito sa pang-araw-araw na pamumuhay. Higit sa lahat, tinumbok din ng pag-aaral ang impluwensyang may malaking impak sa paggamit at pagkabuo ng makabagong salitang balbal.

Mahalaga ang pag-aaral na ito sa pagpapalawak sa pag-unawa ng wika ng mga kabataan at magsisilbing gabay sa pakikitungo sa kanila at pagsabay sa kanilang gamit na wika bilang patunay sa pagtanggap ng pagkakaiba hindi lamang sa katangian at kakayahan pati na rin sa wikang kanilang sinasalita.

\subsection{Layunin ng pag-aaral}

Nilayon ng pag-aaral na mailarawan ang karanasan ng mga partisipante sa paggamit ng balbal na pananalita sa kanilang pang-araw-araw na pakikipagtalastasan at masuri ang (1) nangungunang estruktura sa pagkakabuo ng mga makabagong salitang balbal sa kasalukuyan at (2) ang kadahilanan ng pagsibol ng mga salitang ito. (3) Tinumbok din ng pag-aaral ang impluwensyang may malaking impak sa pagsibol ng mga salitang balbal bilang modernong wika ng henerasyon.

\section{Metodolohiya}

\subsection{Disenyo ng pag-aaral}

Ginamit sa pag-aaral ang Husserlian Descriptive Phenomenology at Colaizzi's Seven Steps of Data Analysis upang mailarawan at masuri ang karanasan ng mga pagrtisipante ukol sa paggamit ng balbal na pananalita sa pamamagitan ng isinagawang pakikipanayam. Ayon nga kay Lester (1999, p. 1), “phenomenology is concerned with the study of experience from the perspective of the individual 'bracketing' taken-for-granted assumptions and usual ways of perceiving. Binanggit naman ni Priest (2004), na ang nanguna sa pagsusulong sa deskriptibong penomenolohiya ay si Edmund Husserl na naglalayong tuklasin, galugarin, at ilarawan ang kahulugan ng isang penomenong naranasan (halaw sa Augusto, 2019). Mabusising hinimay sa pag-aaral na ito ang karanasan ng mga partisipante sa paggamit ng balbal na pananalita kabilang na ang nangungunang estruktura ng pagkabuo ng mga makabagong salitang balbal sa kasalukuyan at ang kadahilan ng pagsibol ng mga salitang ito.Tinumbok din ng pag-aaral ang impluwensyang may malaking impak sa pagsibol ng mga salitang balbal bilang modernong wika ng henerasyon.

Ang mananaliksik ay gumamit ng purposive sampling sa pagpili ng mga partisipante. Ang kriterya ng pamimili ay ang mga sumusunod: (1) kinakailangang nakagamit na siya ng mga makabagong salitang balbal sa pang-araw-araw na pamumuhay; (2) nasa edad 15-19 taong gulang at kasalukuyang nasa Senior High School, at (3) boluntaryong makikilahok bilang partisipante ng pag-aaral. Matapos nito, labintatlong mag-aaral ang napili bilang partisipante ng pag-aaral.

May dalawang bahagi ng instrumento ng pananaliksik - ang unang bahagi ay isang maliit na papel kung saan isusulat ng mga partisipante/informants ang kanilang personal na impormasyon at ang ikalawang bahagi naman ay ang pakikinayam gamit ang limang katanungan tungkol sa mga makabagong salitang balbal na ginagamit, paano ito nabuo at ang kanilang nararamdaman. Ang mga katanungan sa pakikipanayam ay nabuo sa tulong ng isang guro na nagtuturo ng Filipino. Nabuo ang limang katanungan ukol sa pag-usbong ng mga panibagong wikang balbal na ginagamit ng kabataan sa kasalukuyan.

Sa pagusuri ng mga datos ginamit ang pamamaraang nakabatay sa Collaizi's (1978) Seven Steps of Data Analysis. Kinasasangkutan ito ng pitong hakbang (hinalaw sa Gaddi, 2016): (1) Ang mabusising pagsusuri sa mga nakolektang datos ay makatutulong upang magkaroon ng kabuluhan sa mga mananaliksik ang mga natranskrayb na mga datos. (2) Maingat na pagkuha ng mahahalagang pahayag ang isinasagawa; yaong may 
Noval, A. T.

direktang kaugnayan sa penomenon. (3) Bumuo ng mga pagpapakahulugan mula sa pagsusuri sa mga mahahalagang pahayag. Bukod dito, muling binabasa ng mananaliksik ang orihinal na talatanungan na naglalaman ng mga sagot ng mga kalahok. (4)Pagkatapos, bumuo ng mga klaster na makatutulong sa mananaliksik sa pagbuo ng mga tema. (5)Ang mga nabuong tema ay isinama sa mga exhaustive description. Ipagpapatuloy ang balidasyong isasagawa mula sa orihinal na protocol. (6) Pagbabalangkas ng concise na mga pahayag ang bubuoin ng mananaliksik para sa exhaustive descriptions. (7) Balidasyon sa mga nabalangkas o nabuong impormasyon sa pamamagitan ng pagtatanong sa mga sabjek kung may aspekto o bahagi ba ng kanilang karanasaan ang nakaligtaan sa pagsusuring isinagawa.

\subsection{Pinanggalingan ng mga datos}

Ang mananaliksik ay nagsagawa ng pakikipanayam sa labintatlong nakapasang partisipante na mga mag-aaral ng Senior High School. Sa pagsasagawa ng pakikipanayam, isinaalang-alang ng mananaliksik ang etikal na tuntunin sa pagsasagawa ng pananaliksik sa pamamagitan ng isang liham pahintulot para sa mga partisipante. Ipinaliwanag din ng mananaliksik sa mga partisipante ang mga layunin na ninais matamo sa pag-aaral. Tiniyak din ng mananaliksik na mananatiling confidential ang kanilang mga pagkakakilanlan sa pamamagitan ng paggamit ng mga koda at ang lahat ng mga datos na nalikom ay gagamitin lamang sang-ayon sa mga layunin ng pag-aaral. Humingi ng pahintulot sa mga partisipante sa paggamit ng recorder sa pagsasagawa ng pakikipanayam. Matapos maisagawa ang pakikipanayam, pinakinggan at itinala ng mananaliksik ang nairekord na mga sagot sa pakikipanayam. Batay sa nilalaman ng linya ng mga pahayag ng mga partisipante, sinuri ng mananaliksik ang mga pahayag at bumuo ng tema o kategorya.

Ang kasunod na hakbang ay ang pagsusuri sa tugon ng mga partisipante ayon sa mga hakbang na inilahad sa Colaizzi's Seven Steps of Data Analysis. Una, binasa ng mananaliksik ang lahat ng deskripyon ng mga protokol. Sinundan naman ito sa pagkuha ng mga mahahalagang pahayag mula sa tugon ng mga partisipante ukol sa kanilang mga karanasan sa paggamit ng mga balbal na pananalita at pinagbigay rin sila ng mga halimbawang salitang balbal. Pagkatapos, binigyan ng pagpapakahulugan ang mga mahahalagang pahayag na lutang na lutang sa mga tugon na nalikom. Sinundan naman ito ng pagsasaayos ng mga nabuong pagpapakahulugan sa pamamagitan ng pagkaklaster ng mga tema na may kaugnayan sa mga dahilan sa pagsibol ng mga makabagong salitang balbal sa modernong panahon, paraan ng pagkabuo ng mga salitang balbal sa kasalukuyan, at impluwensyang may malaking impak sa pagkakabuo ng mga salitang balbal. Matapos na mabuo ang klaster ng mga tema, binalangkas ng mananaliksik ang kabuoang paglalarawan sa penomenong pinag-aaralan. Panghuli, sa pagbabalideyt ng pangkalahatang pag-aaral, binalikan ng mananaliksik ang mga partisipante at tinanong sila sa kaangkupan ng naging kinalabasan ng pag-aaral sa kanilang naging karansan. Hiningan din ng komento at suhestiyon ang mga kalahok upang mabigyang-linaw ang kabuoang pag-aaral.

\subsection{Limitasyon ng pag-aaral}

Sa pag-aanalisa, hinimay ang mga pahayag o tugon ng mga partisipante sa isinagawang pakikipanayam sa labintatlong (13) mag-aaral ng SHS. Matapos nito, itinala ang mga balbal na pananalita, ang estruktura ng pagkakabuo nito at tiningnan ang pagkakahawig at relasyon ng mga tugon at bumuo ng mga temang may kaangkupan sa mga pahayag. Nakatuon ang paglalarawan sa karanasan ng mga partisipante sa paggamit ng balbal na pananalita sa kasalukuyang panahon at masuri ang nangungunang estruktura sa pagkakabuo ng mga makabagong salitang balbal sa kasalukuyan at mga kadahilanan ng pagsibol ng mga salitang ito. Tinumbok din ng pag-aaral ang impluwensyang may malaking impak sa pagsibol ng mga salitang balbal bilang modernong wika ng henerasyon.

\section{Resulta at pagtatalakay}

Inilalahad sa bahaging ito ang pagsusuri sa tugon ng mga partisipante batay sa mga sumusunod: pagtatala ng mga salitang balbal na pananalitang ginamit ng mga partisipante, kinalabasan ng pakikipanayam ukol sa mga

4 Consortia Academia Publishing (A partner of Network of Professional Researchers and Educators) 
Pag-usbong ng balbal na pananalita bilang modernong wika ng kabataan: Isang pagsusuri

dahilan at impluwensya ng paggamit nito. Ang mga tugon ay pinagpangkat-pangkat ang mga konseptong nakuha mula sa pakikipanayam at isinaayos ayon sa kategorya o tema.

\subsection{Estruktura ng pagkabuo ng mga makabagong salitang balbal}

Inilalahad sa bahaging ito ang pagsusuri sa paraan at estruktura ng mga makabagong salitang balbal na nabubuo sa kasalukuyang panahon. Sinuri at tinukoy ang klasipikasyon ng pamaraan at estruktura ng mga makabagong salitang balbal at binigyan ito ng pagpapakahulugan.

\section{Talahanayan 1}

Paraan at estruktura ng pagkabuo ng mga makabagong salitang balbal sa kasalukuyan

\begin{tabular}{|c|c|c|}
\hline Estruktura ng Pagkabuo ng Salitang Balbal & Mga Salita & Kahulugan \\
\hline \multirow[t]{9}{*}{ Pagbabaligtad } & lodi $(\mathrm{P} 1,2,6,7,11)$ & idol \\
\hline & erpat $(\mathrm{P} 3)$ & father \\
\hline & werpa $(\mathrm{P} 5,6)$ & power \\
\hline & abab (P5) & baba/bibig \\
\hline & nosi (P6) & sino \\
\hline & petmalu $(\mathrm{P} 5,6,7,11,13)$ & malupet \\
\hline & bayu (P11) & uyab/kasintahan \\
\hline & imal (P9) & lami/masarap \\
\hline & yeko ar (P10) & okey lang \\
\hline \multirow[t]{4}{*}{ Paggamit ng Akronim } & SML $(\mathrm{P} 3,7,13)$ & share mo lang \\
\hline & SKL (P7) & share ko lang \\
\hline & LOL (P9) & laugh out loud \\
\hline & P.S. (P13) & pahabol na salita \\
\hline \multirow[t]{4}{*}{ Pagpapalit-pantig } & jowa $(\mathrm{P} 8)$ & asawa \\
\hline & juntis (P8) & buntis \\
\hline & shofo $(\mathrm{P} 6,9)$ & gwapo \\
\hline & shufa $(\mathrm{P} 7,9)$ & gwapa/maganda \\
\hline \multirow[t]{18}{*}{ Kombinasyon } & angatch $(\mathrm{P} 1,11)$ & tanga/laging sablay \\
\hline & gora $(\mathrm{P} 1,2,12)$ & tara na \\
\hline & sinetch $(\mathrm{P} 1)$ & sino \\
\hline & charlangs (P1) & joke lang \\
\hline & aketch (P1) & ako \\
\hline & beshy (P1,2,7,11,) & best friend \\
\hline & shudi abas $(\mathrm{P} 2,5,10)$ & wag mag-ingay \\
\hline & aw na dutch $(\mathrm{P} 2,5,10)$ & walang pera \\
\hline & nanatch $(\mathrm{P} 2)$ & lahat \\
\hline & yadz $(\mathrm{P} 3,7,12)$ & babae \\
\hline & siszum (P3) & kapatid na babae \\
\hline & gorabels (P4,11) & tara na \\
\hline & gotch na oks (P4) & tulog na ako \\
\hline & ikal $(\mathrm{P} 4,12)$ & lalaki \\
\hline & mudrakels (P4) & nanay \\
\hline & siszt $(\mathrm{P} 4,5)$ & kapatid babae \\
\hline & mars (P6) & kumare \\
\hline & shookt (P8) & takot \\
\hline
\end{tabular}

Inilalahad sa talahanayan 1 na ang pamaraan at estruktura ng mga salitang balbal sa kasalukuyan ay klasipikasyon na pagbabaligtad, paggamit ng akronim, pagpapalit pantig at kombinasyon.

Ipinalulutang ng talahanayan 1 na ang estrukturang pabaligtad ay kinabibilangan ng mga salitang lodi, erpat, werpa, abab, nosi, petmalu, bayu, imal at yeko ar. Sa kabilang banda ang mga salitang SML, SKL, LOL at P.S. ay maikaklasipika sa paggamit ng akronim. Samatala, ang mga salitang jowa, juntis, shofo at shufa naman ay mauuri pagpapalit pantig na mga salita habang ang mga salitang angatch, gora, sinetch, charlangs, aketch, beshy, shudi abas, aw na dutch, nanatch, yadz, siszum, gorabels, gotch na oks, ikal, mudrakels, siszt, mars at shookt naman ay nasa estrukturang kombinasyon. Sa madaling salita, ang nangungunang paraan ng pagkakabuo ng mga 
Noval, A. T.

salitang balbal sa kasalukuyan ay sa pamamagitan ng kombinasyon.

Ang kumbinasyong pamaraan ng pagkabuo ng mga salitang balbal ay sa pamamagitan ng pagbabaligtad at pagdaragdag, pagpapaikli at pagdaragdag, pagpapaikli at pagbabaligtad, panghihiram at pagpapaikli at panghihiram at pagdaragdag. Kaugnay nito, binanggit din nila na ang salitang balbal ang pinakadinamiko sa lahat ng antas ng wika sa kadahilanang ang usong salita sa kasalukuyan ay malalaos o mapapalitan naman sa madaling panahon (Aldaca at Villarin, 2012).

Sa pag-aaral ni Francisco (2014), binanggit niya na ang lahat ng mga salitang balbal ay may kanya-kanyang konteksto ng pagkakalikha. Tanging ang mga nabubuong social fragment ang makalilikha ng salita na magkaklasipika dito at tanging ang koleksyon ng mga social fragment ay maaaring magresulta ng enunsasyong diskurso na may kaugnayan sa kasalukuyan at kongkretong sistema.

Ayon naman kay Mutunda (2007), na ang kadalasang pamamaraan ng mga pagbabagong naganap sa mga salitang balbal sa Nyanja at iba pang anyo nito. Ilan sa mga salitang balbal ay mula sa mga naunang mga salita. Dagdag nito, ang deskripsyon ng mga salitang balbal sa Nyanja ay hindi exhaustive dahil ang wika ay dinamiko at tulad ng ibang kabataan saanmang sulok ng mundo, ang kabataang Luska ay patuloy na lilikha ng panibagong bokabularyong balbal.

Pinatutunayan lamang sa pag-aaral nina Francisco (2014) at Mutunda (2007) na bagamat ang salitang balbal ay pinaniniwalang sinasalita ng maliit na yunit ng lipunan at sumisibol sang-ayon sa pangangailangan ng sitwasyong pangwika, kakikitaan naman ito ng mga estruktura ng pagkakabuo.

Bilang paglalahat, mahihinuha sa mga kaugnay na pag-aaral na binanggit na ang kabataan sa kasalukuyang henerasyon ay may sariling wikang gamit. Ang mga wikang ito ay ginagamit nila sang-ayon sa pangangailang dulot ng kanilang lipunan at bunga na rin ng kanilang malawak na pag-iisip. Sa patuloy na pakikipagkapwa ng makabagong henerasyon, nakabubuo ng wikang natatangi sa kanilang henerasyon na makatutulong upang maipahayag ang kanilang mga sarili.

\subsection{Mga tema o kategoryang nabuo sa ginawang pagsusuri}

Ang deskriptibong pagsusuri sa isinagawang pakikipanayam ay nagbigay-daan sa pagtumbok ng anim (6) na temang bumabalot sa sagot ng mga partisipante (P). Ang mga pahayag na isinagot ng mga partisipante ay nilagyan ng mga panipi upang ipahayag ang orihinal na tugong ibinahagi ng partisipante. Ang mga temang nabuo batay sa mga tugon ng mga partisipante $(\mathrm{P})$ ay ang mga sumusunod:

Tema 1: Libangan at Katuwaan o Kasiyahan

Tema 2: Pagpapahayag o Sariling Pagkakakilanlan

Tema 3: Pagsabay sa Uso o Trend at Pakikisalamuha o Pakikisama

Tema 4: Pagkamalikhain

Tema 5: Pagpapaikli, Pagbabali-baliktad at Pagbabagong-Ayos ng mga Salita

Tema 6: Impluwensya ng Lipunan

\subsection{Mga dahilan sa pagsibol ng mga makabagong salitang balbal sa modernong panahon}

Ang mga partisipante (P) ng pag-aaral ay tinanong kung ano ang kanilang nararamdaman at ang mga dahilan ng paggamit ng mga makabagong salitang balbal na umuusbong sa kasalukuyan.

\section{Tema 1 Libangan at Katuwaan o Kasiyahan}

"Ako po ay natutuwa at nalilibang sa mga salitang iyon kasi parang ito po ay makabago at hindi ko ito kadalasang naririnig sa mga taong aking nakakasalamuha." (P1)

"Uhm...parang natutuwa lang ako...ah na-eenjoy ko yun kasi naka... nakasanayan ko na kasing gamitin." (P3)

6 Consortia Academia Publishing (A partner of Network of Professional Researchers and Educators) 
Pag-usbong ng balbal na pananalita bilang modernong wika ng kabataan: Isang pagsusuri

"Uhm... ang nararam. Nararamdaman ko kapag ginagamit ko yung mga salitang ito ay nasisiyahan ako sir at uhm... ang ah... yun po nasisiyahan.” (P4)

"Para po sa akin, ako ay nasisiyahan o natutuwa" (P7)

Tema 2 Pagpapahayag o Sariling Pagkakakilanlan

"Dahil na rin sa pabago-bago ang sistema natin so marami na ring nagbago na mga salita kaga dahil na rin sa mga dahil na rin sa teknolohiya na nabubuo may marami nag at sa mga... mga... mga... kagaya lamang kagaya dahil sa social media so nandun pwede natin i-express yun so parang... nabuo ang kung ano-ano nalang salita na pinagbago-bago na.” (P12)

"Para sa akin po sir, dahil ah may mga tao kasi na uhm grupo-grupo na hindi nila alam na ano ang ay may gusto... kanang gusto rin nila sila lang yun ang makakaalam kung ano ang sinasabi nila kaya nabuo ito upang mas nakata... ay maitago nila ito sa ibang tao...” (P13)

Tema 3 Pagsabay sa Uso o Trend at Pakikisalamuha o Pakikisama

"Sa tingin ko lang sir, dahil... dahlan ito sa pagiging mga siguro yung mga barkada nila then nag-ano sila nakikiuso lang sa mga kabataan.” (P2)

"Uhm... isa sa mga nararamdaman ko is "pag binibigkas ko ito is awkward para sa akin kasi parang... parang ano hindi pa ako sanay magsalita pero na-fefeel ko na isa ako sa mga "in" ngayon na mga yung parang naka... nakakasabay ako kung ano ang uso ngayon.”(P5)

“Uhm... para lang po sa akin sir, para hndi po mahuli sa uso at para hindi maging ignorante” (P8)

“Uhm... marahil siguro dahil uso po ito" (P10)

Ginagamit ang mga makabagong salitang balbal sa patuloy na pagpapahayag ng tao sa kanyang lipunan bilang paraan ng pakikisalamuha o pakikisama. Sa patuloy na pakikipag-ugnayan ng tao kasabay ang pagbabago ng panahon ay ginagamit ang mga salitang balbal at naging bahagi na ito sa pang-araw-araw na pakikipagtalastasan.

Ayon naman kay Güniz (2015), “Tanggapin man natin o hindi ang wikang balbal ay nakaimpluwensya sa wikang Ingles. Kinalimutan natin ang pagkakaiba ng pormal at impormal na piyesa ng pagsulat at ginamit natin ang mga salitang balbal. Dumarami ang mga taong nagsisimula at nakauunawa sa mga wikang balbal. Kung kaya ang salitang balbal ay hindi lamang wika, diyalekto o ordinaryong rejister. Ito ay isang espesyal na koda na naiiba sa gumagamit nito, gumagawa ng eksena. Ipinapakahulugan na ang eksena ay kultura ng kabataan. Ang mga salitang balbal ay hindi isang profanity o walang barila. May mga salitang balbal para sa droga at pagtatalik ngunit ito ay dahil sa paksang kinagustuhan ng kabataan." Sa madaling salita, ang mga wikang balbal ng modernong panahon ay may mahalagang gampanin sa diskurso ng mga kabataan sa modernong panahon. Hindi lamang ito nabubuo ng basta-basta lang kundi may mga dahilan sa pagkakabuo nito.

Sa pag-aaral nina Abel et al, (2016), binanggit nila ang mga bagong salita at pagpapakahulugang nalinang ng mga Pilipinong manlalaro ay simbolo ng pagkakakilanlan ng kalagayan ng lipunan sa Pilipinas sa pamamagitan ng pagkilala kung paano ito ginagamit sa pakikipagtalastasan. Kaugnay nito, ang komunidad ng mga manlalarong Pilipino ay itinuturing na toxic ngunit dahil sa larong ito, ang dinamikong interaksyon at komunikasyon ng mga manlalaro ay nabubuo anuman ang kalalabasan ng kanilang laro.

Bilang paglalahat, masasabing ang mga salitang balbal sa makabagong henerasyon ay mahalagang sangkap bilang diskurso ng kabataan. Ginagamit ang mga makabagong salitang balbal bilang kaakuhan at sa patuloy na pagpapahayag ng kabataan sa lipunan bilang paraan ng pakikisalamuha o pakikisama. Sa pamamagitan nito, 
Noval, A. T.

naipaaabot niya ang kanyang mga ninanais, ideya at saloobin. Higit sa lahat, nakatutulong din ang mga salitang ito upang makasabay sa kung anoman ang tampok nang sa gayon ay maramdaman ng kabataan na sila ay kabahagi at hindi nalalayo sa kanilang henerasyon.

3.4 Paraan ng pagkabuo ng mga salitang balbal sa kasalukuyang panahon

Ang mga partisipante $(\mathrm{P}) \mathrm{ng}$ pag-aaral ay tinanong tungkol sa kanilang nalalaman sa dahilan ng pagkakabuo ng mga makabagong salitang balbal sa kasalukuyang panahon.

\section{Tema 4 Pagkamalikhain}

"Para sa akin, nabuo yung ganung klase ng pananalita dahil na rin sa lawak ng pag-iisip ng tao. Nakalilikha tayo ng mga malilikhaing... malilikhaing bagay at mga pananalita na magagamit natin sa pang-araw-araw na buhay.” (P3)

"Uhm... para sa akin, nabuo ang mga ganitong pagsasalita 'pag mga salita is dahil sa palitan ng pag-iisip natin dahil sa pagiging creative ng mga tao kaya tayo nakbubuo ng ganitong klaseng salita." (P5)

"Siguro po kasi ang mga kabataang millennial ay yung creative, malikhain so ganaha... so rin po nilang makapag-imbento ng mga bagong salita kung saan mas siguro mas makaintindi sila kung ewan ko ba kung nakaintindi sila ng ganun or sadyang yah something they want to be creative lang talaga na makapaglikha ng mga bagong salita." (P9)

\section{Tema 5 Pagpapaikli, Pagbabali-baliktad at Pagbabagong-Ayos ng mga Salita}

"Ah... ang nararamdaman ko po sir parang ok lang pero 'pag inisip mo na tinanong mo sarili mo kung bakit ba... bakit ba kailangan nilang... kailangan nilang bali-baliktarin kung meron namang... namang madaling salita." (P2)

"Para po sa akin, nabubuo ito sa isipan ng tao kasi kagaya ng sinabi ko pinaikli.” (P6)

"Uhm... sa una na nakapag-isip po ako bakit ba ito binabaliktad ano bang dahilan nila para baliktarin ito at napag-iisipan kop o na siguro millennial ako kaya kailangan na siguro akong makisabay kung ano ang uso." (P9)

"Siguro dahil po sa sosyal... sosyal media po kasi nakikita ko po dun may mag-popost po na hindi po... hindi po a ano po kanang bago po sa akin na masasabi na... parang masasabi mo 'anong anong ibig sabihin nito' ganun po tapos malalaman mo rin ganun pala binaliktad lang pala nila." (P11)

Nabubuo ang mga makabagong salitang balbal dulot ng pagkamalikhain ng tao sa pagpapaikli, pagbabaliktad at pagbabagong ayos ng mga salita sa layuning maipaabot ang kanyang mga ideya at saloobin. Sa lawak ng pag-iisip at pagkamalikhain ng tao nabubuo ang mga makabagong salitang balbal alinsunod sa pangangailangan ng tao sa pagpapahayag. Ang kagalingang taglay na ito ng isang tao sa paggamit ng wika ay ang pangunahing dahilan ng pagkakaroon ng kaantasan ng wika. Ang paghahalo-halo ng wika sa isa pang wika, panghihiram, pagbabaliktad atbpa. ay halimbawa ng kaantasan ng wika.

Isang mahalagang katangian ng wika ay ang pagkakaroon nito ng antas. May iba't ibang kategoryang kinabibilangan na naayon sa klasipikasyon ng antas nito. Ang antas ng wika ay kadalasang may kaugnayan sa katayuan sa lipunan ng taong gumagamit nito (Dumapias, 2018).

Sa teoryang sosyolinggwistiks pinaniniwalaan na ang wika ay hindi isang simpleng komunikasyon na ginagamit ng indibidwal ayon sa isang sistema ng mga alituntunin kundi isang kolektibong pwersa, isang

8 Consortia Academia Publishing (A partner of Network of Professional Researchers and Educators) 
Pag-usbong ng balbal na pananalita bilang modernong wika ng kabataan: Isang pagsusuri

pagsasama-sama ng mga anyo sa isang magkaibang kultural at sosyal na mga gawain at grupo (Alcaraz, 2016).

Binanggit sa pag-aaral nina Oco et al. (2015), napag-alaman na ang resorses, isyu, pagsubok at kompyutisyunal na karakteristik ay madetalyado. Natuklasan sa pag-aaral na ito na ang karamihan sa mga pananalitang beki ay hindi saklaw ng rule file at hindi makikita sa mga ginagamit na resorses. Bilang isang sosyolek na naging bahagi na ng lipunan, kinakailangan ang constant na pag-update sa pagbabago at pag-usbong ng mga pananalitang ito.

Bilang paglalahat, nabubuo ang mga makabagong salitang balbal dulot ng pagkamalikhain ng tao sa pagpapaikli, pagbabaliktad at pagbabagong ayos ng mga salita sa layuning maipaabot ang kanyang mga ideya at saloobin. Patunay lamang ito na ang bawat tagapagsalita ay may kanya-kanyang estilo at paraan ng pagpapahayag. Sa ganitong paraan, napapalawak ang midyum na ginagamit upang mas lalong maipahayag ang mensahe sa pamaraan na komportable ang tagapagsalita nito.

\subsection{Impluwensyang may malaking impak sa pagkakabuo ng mga salitang balbal}

Ang mga partisipante (P) ng pag-aaral ay tinanong tungkol sa impluwensyang may malaking impak sa kanila upang gamitin ang mga salitang balbal sa pakikipagtalastasan. Ang mga tugon sa ibaba ang kanilang naging tugon ukol sa katanungang ito:

\section{Tema 6 Impluwensya ng Lipunan}

"Para po... sa ngayon kapag nanood po ako ng "It's Showtime” yung si Vice Ganda siya pong naging influence sa ngayon kay dahil kanang... marami po siyang kanang... na-entertain po siya kanang... na-entertain po siya sa amin yung mga kabataan lalong-lalo na po sa mga tao na idolo po siya.” (P7)

"Yung mga taong nasa paligid kasi sila rin po nakikiuso rin po kasi sila ah mara... marami ah... madalas po sila rin po nilang ginagamit yung mga salitang 'yan.” (P10)

"Para sa akin na kung ano kasi kanang kung ano kasi ang nandito tayo sa makabagong panahon so kung anong nakikita natin sa telebisyon, social media so parang nakukuha ang atensyon natin so tayo parang nadadala na rin natin 'yan yung mga kaibigan mo magsasalita ng mga ganyan so nadadala... oo.” (P12)

May malaking impak sa kabataang milinyal ang teknolohiya, social media, mga kaibigan at iniidolong artista sa paggamit ng mga makabagong salitang balbal sa pang-araw-araw na pamumuhay. Ang impluwensyang panlipunan ay may malaking impak sa kabataang milinyal sa paggamit ng mga makabagong salitang balbal sa pakikipagtalastasan.

Ang social media ay isang makabagong teknolohiya na nagbibigay-daan sa mga tao sa iba't ibang panig ng mundo na magkaroon ng koneksyon, makipag-ugnayan, bumuo at magbahagi ng impormasyon (Lewis, 2010). Dagdag naman nina Howard at Parks (2012), ito rin ay isang mahalagang sangkap ng komunikasyon (halaw sa Tubio et al., 2017). Sa pamamagitan ng social media, nabibigyan ng malawak na midyum ang tao upang maipahayag ang sarili - hinaing, kaligayahan, pagmamahal, kabiguan at iba pang damdamin nararanasan sa kanyang mga kaibigan, kaklase, kapamilya at maging sa mga taong hindi kakilala. Ito marahil ang dahilan ng pagsibol ng mga makabagong salitang balbal na karaniwang ginagamit ng mga kabataan sa kasalukuyan. Dahil dito, naging talamak ang paggamit ng mga salitang ito bilang bahagi ng pagpapahayag ng kabataan sa kanyang lipunan.

Dagdag nito, ayon kay Barnett (2011) na sa pamamagitan ng social media, nabubuo ang isang kasangkapang magsisilbing daan sa mga gumagamit nito na makipagtalastasan sa kanilang online networks sa lahat ng pagkakataon, pinagsama ang kanilang pisikal at online na presensya, inaangat nito ang larangan ng makabagong 
Noval, A. T.

uri ng komunikasyon (halaw sa Abadiano et. al., 2014).

Natuklasan sa pag-aaral nina Tubio et al. (2017) na maraming mga salita ang umiiral sa social media sa kasalukuyang panahon at karamihan dito ay ngayon pa lamang narinig dahil na rin sa pag-usbong ng makabagong panahon. Maraming mag-aaral ang nagsasabi na ang mga salitang ito ay mahalaga para sa kanila at natutulungan sila nito para mapadali ang pakikipag-usap sa ibang tao ngunit karamihan sa mga salitang ito ay hindi nagugustuhan at nauunawaan ng ibang mag-aaral.

Bilang paglalahat, malaki ang impak ng mga kaibigan at iniidolong artista bilang impluwensya sa paggamit ng mga makabagong salitang balbal sa pang-araw-araw na pamumuhay. Hayagan nitong isinasaad na mabisang kasangkapan ang mga ito sa pagsagap ng mga kabataang milinyal ang teknolohiya, social media, impormasyong makaiimpluwensya sa pagkatao at higit sa lahat sa paraan ng pakikipagkomunikasyon.

\section{Kongklusyon at rekomendasyon}

Nilayon ng pag-aaral na masuri ang nangungunang estruktura sa pagkakabuo ng mga makabagong salitang balbal sa kasalukuyan at ang kadahilanan ng pagsibol ng mga salitang ito. Tinumbok din ng pag-aaral ang impluwensyang may malaking impak sa pagsibol ng mga salitang balbal bilang modernong wika ng henerasyon. Ang nangungunang paraan sa pagkakabuo ng salitang balbal sa makabagong henerasyon ay sa pamamagitan ng kombinasyon. Ginagamit ng mga kabataan sa kasalukuyan ang mga makabagong salitang balbal bilang libangan at pakikisama o pakikisalamuha sa pamamagitan ng malikhaing pagpapaikli, pagbabaliktad at pagbabagong ayos ng mga salita sa pakikipagtalastasan. Malaki ang impluwensya ng teknolohiya, social media, mga kaibigan at iniidolong artista sa paggamit ng mga ganitong uri ng pananalita.

Sa kasalukuyang panahon, mahalagang aspekto ng pakikisalamuha o pakikisama ay ang pagsabay sa uso o anomang trending. Sa larangan ng pagpapahayag, ang paggamit ng makabagong salitang balbal ay isang paraan sa pagsabay sa daloy ng panahon. Sa taglay na lawak na pag-iisip ng tao dala ng pagkamalikhain, nakatuklas ang tao ng ibang pamamaraan ng pagpapahayag. Maaaring ang pamamaraang ito ay sa pamamagitan ng pagpapaikli, pagbabaliktad at pagbabagong ayos ng mga salita. Sa ganitong paraan, lubusang napapahayag ng tao ang kanyang sarili sa natatangi at kakaibang pamamaraan. Napakalaki ng impluwensya ng teknolohiya, social media, mga kaibigan at iniidolong artista sa mga kabataang milinyal sa paggamit ng ganitong uri ng pananalita. Ang panggagaya at pagsunod sa kanilang nakikita, naririnig at iba pang karanasan sa pagsasalita ay nagpaparamdam sa kanila na sila ay kabilang at nakakasabay sa kanilang henerasyon.

Napapanahon ang pag-aaral na ito dahil na rin sa impluwensiya ng social media at teknolohiya sa wikang gamit ng mga mag-aaral higit lalo sa paggamit ng mga balbal na pananalita. Kung kaya mahalagang mapag-aralan ang mga ito nang sa gayon ay maunawaan ng mga guro ang wikang gamit ng kanilang mga mag-aaral sa ganitong paraan ang komunikasyon ng guro sa kanyang tinuturuan ay hindi mahahadlangan sapagkat mayroon siyang sapat na kaalaman sa pag-unawa sa mga salitang ito. Napapanahon din na mapag-aralan ang pagkamalikhain ng mga mag-aaral sa paggamit ng wika upang mas malinang pa ang kanilang kahusayan pagdating sa kasanayan sa pagsasalita sang-ayon sa mga layunin na kinakailangang linangin sa makrong kasanayang ito.

Inirerekomenda ng mananaliksik na magsagawa pa ng ibayong pag-aaral sa mga wikang ginagamit ng kabataan dahil sa mabilis magbago ang wika ayon sa pangangailan ng kanilang henerasyon. Magkaroon ng bukas na isipan sa pag-unawa sa kabataan dahil ang pag-unawa sa kanilang wika ay pagkilala sa kanilang mga natatanging kakayahan at pagkatao. Gamitin sa pagtuturo ang mga salita ng kabataan bilang ambag sa pagpapayaman ng diskursong Pilipino.

Acknowledgement - Bahagi ng papel na ito ay naipresenta sa $6^{\text {th }}$ International Teacher Education Student 
Pag-usbong ng balbal na pananalita bilang modernong wika ng kabataan: Isang pagsusuri

Conference (TESTCOn) na may temang "Futuristic Multidisciplinary Research Across Borders" noong April 12, 2019 sa Teaching Arts Centrum, Cebu Normal University, Cebu City, Philipines.

\section{Mga sanggunian}

Abadiano, M. N., Bonotan, A., \& Makiling R. (2014). The dynamics of netizens' infraction sharing in social media: why do we see information in social media? International Journal of Interdisciplinary Research and Innovations, 2(3), 30-55.

Abel, M., Autor, C., Gripal, A., \& Demetrio III, F. (2016). The language of Filipino gamers: analysis of the origin and meaning of words being used in the worlds of Dota 2 and Lol [Wika ng mga manlalarong Pilipino: pagsusuri sa pinagmulan at saysay ng mga salitang ginagamit sa mundo ng Dota 2 at LoL]. Malay, 29(1), 45-68.

Alcaraz, C.V., Jocson, M. O., \& Villafuerte, P. V. (2016). Communication and research for senior high school [Komunikasyon at pananaliksik para sa senior high school]. Educational Resources Corporation.

Aldaca, B. B. \& Villarin, I. J. (2012). Communication in Filipino academics: reference and book of exercises in Filipino 1, tertiary level [Komunikasyon sa akademikong Filipino: batayan at sanayang-aklat sa Filipino 1, antas-tersyarya]. Maxcor Publishing House Inc.

Augusto, W. S., Jr. (2019). A phenomenological study on the lived experience of the out-of-field mentors. International Journal of Advance Research and Publications, 3(6), 35-42.

Dumapias, L. M. L. (2018). Daksigya: communication and research in language and Filipino culture [Daksigya: komunikasyon at pananaliksik sa wika at kulturang Pilipino]. CMU Press.

Estanislao, J.C., Padrigan, J. C., Cabanes, R. V., Lopez, K. J., Pallega, R. J., Ituralde, J., Tapar, J., \& Pacat, J. (2018). Effects of slang on the learning of selected grade 12 students in ABM strand this school year [Epekto ng salitang balbal sa pag-aaral ng mga piling mag-aaral sa ikalabin-dalawang baitang sa strand na ABM panuruang taon] [Tesis]. https://www.academia.edu/36190864/EPEKTO_NG_SALITANG_BALBAL_SA_PAG-AARAL_NG_ MGA_PILING_MAG_AARAL_SA_IKALABING_DALAWANG_BAITANG_SA_STRAND_NA_A BM_PANURUANG_TAONG_2018

Francisco, L. (2014). Heteronormativity: good language and bad slang: sex and gender roles of Brazilian traversities in the context of transnational prostitution. New University of Lisbon.

Gaddi, Z.A. (2016). From experiences to themes: phenomenology on the school's culture contributing to Eexcellence. AsTEN JOURNAL of Teacher Education, 1(2), 99-110.

Güniz Çelik, (2015). Slang language movement. https://www.academia.edu/21474986/Topic_Slang_Language_Movement

Lester, S. (1999). An introduction to phenomenological research. https://www.researchgate.net/publication/255647619_An_intoroduction_to_phenomenological_researc $\mathrm{h}$

Mutunda, S. (2007). Language behavior in Lusaka: the use of Nyanja slang. The International Journal of Language Society and Culture. https://www.researchgate.net/publication/251622306_Language_Behavior_in_Lusaka_The_Use_of_Ny anja_Slang

Oco, N., Fajutagana, C. M. L., Miñon, J. D., Morano, J. A., \& Tinoco, R. C. (2015). Witchebelles anata magcharot kay mudra na nagsusuba si akech: developing a rule-based unidirectional beki lingo to Filipino translator. https://www.national-u.edu.ph/wp-content/uploads/2016/08/Witchebelles-Anata-Magcharot-kay-Mudra -na-Nagsusuba-si-Akech-Developing-a-Rule-based-Unidirectional-Beki-Lingo-to-Filipino-Translator.p $\underline{\mathrm{df}}$

Quia, L. J. M., Rivera, N. A. R., Rustia, J. E. R., Tinio, M. J. L., \& Varona, A. P. (2018). Study on the impact of the use of slang on selected students of 11 ABM [Pag-aaral tungkol sa epekto ng paggamit ng mga 
Noval, A. T.

salitang balbal sa piling mag-aaral ng $11 \mathrm{ABM}$ ] [Tesis, University of Perpetual Help System Dalta].

Tubio, A. Y., Platilla, A. P., Ancheta, J. M. O., Oaña, J. R. L., Corre, J. R. J., Ochave, J. A., \& Matunog, N. B. P. (2017). Perception of grade 11 students in selected schools in Trece Martires City about the language existing on social media [Pananaw ng mga mag-aaral sa baitang 11 sa mga piling paaralan sa Trece Martires City hinggil sa umiiral na salita sa social media] [Tesis, Trece Martires Senior High School, Cavite]. 